\title{
Transesophageal pulmonary nodule biopsy using endoscopic ultrasonography
}

\author{
Basil S. Nasir, MBBCh, Marcel Edwards, MD, Vicky Tiffault, RN, Jordan Kazakov, MD, \\ Mohammed Khereba, MD, Pasquale Ferraro, MD, and Moishe Liberman, MD, PhD
}

\begin{abstract}
Objective: Parenchymal pulmonary nodules located in proximity to the mediastinum, vertebral column, major vessels, or behind the heart can be technically challenging and dangerous to biopsy using traditional image-guided techniques. Endoscopic ultrasound-guided fine needle aspiration (EUS-FNA) can be used to access some of these difficult to reach lesions. The purpose of the present study was to report our experience with this technique in a consecutive cohort of selected patients.
\end{abstract}

\begin{abstract}
Methods: This was a retrospective cohort study. Eligible patients were identified from a prospective database. A transesophageal approach under real-time EUS guidance was performed using a 22-gauge needle. All patients underwent postprocedural chest radiography and were followed up at 30 days.

Results: During a 31-month period, 55 patients underwent EUS-guided lung biopsy. Confirmatory visual correlation of nodule localization within the lung parenchyma between computed tomography and EUS was possible in $100 \%$ of cases. The lung nodule distribution was $41.5 \%$ right upper lung, $18.9 \%$ right lower lung, $28.3 \%$ left upper lung, and $11.3 \%$ left lower lung. Histologic and cytologic sampling was adequate in 52 of the 55 procedures $(94.5 \%)$. In all patients with adequate biopsy sampling, accurate pathocytologic diagnoses of the target parenchymal nodules were obtained. The accuracy and sensitivity of EUS-FNA were both $94.5 \%$ and consistent with the diagnosis on pathologic resection or clinical progression of disease, or both. No morbidity resulted from the procedure nor was observed at 30 days.
\end{abstract}

Conclusions: EUS-FNA of parenchymal pulmonary nodules is safe and accurate and allows for biopsy of perimediastinal lung lesions not attainable using traditional techniques. (J Thorac Cardiovasc Surg 2014;148:850-5)

Lung cancer is the leading cause of cancer death in the United States, with an estimated incidence of 224,210 new cases in 2014. ${ }^{1}$ Patients with non-small cell lung cancer (NSCLC) typically present with a lung nodule or mass that might or might not be symptomatic. Recently, the National Lung Screening Trial has shown that screening with low-dose computed tomography (CT) is efficacious and associated with reduced mortality. ${ }^{2}$ Thus, lung cancer screening is expected to increase the number of patients presenting for diagnostic evaluation of a pulmonary nodule or mass. In certain patients, tissue for pathologic analysis is required to rule out or rule in malignancy.

From the CHUM Endoscopic Tracheo-bronchial and Oesophageal Center, Division of Thoracic Surgery, Department of Surgery, University of Montréal, Montréal, Québec, Canada.

Disclosures: Authors have nothing to disclose with regard to commercial support.

Read at the 94th Annual Meeting of The American Association for Thoracic Surgery, Toronto, Ontario, Canada, April 26-30, 2014.

Received for publication April 9, 2014; revisions received May 26, 2014; accepted for publication June 2, 2014; available ahead of print July 18, 2014

Address for reprints: Moishe Liberman, MD, PhD, CHUM Endoscopic Tracheobronchial and Oesophageal Center, Division of Thoracic Surgery, Department of Surgery, Centre Hospitalier de l'Université de Montréal, 1560 rue Sherbrooke Est, 8e CD - Pavillon Lachapelle, Bureau D-8051 Montréal, Québec H2L 4M1, Canada (E-mail: moishe.liberman@umontreal.ca).

0022-5223/\$36.00

Copyright (c) 2014 by The American Association for Thoracic Surgery

http://dx.doi.org/10.1016/j.jtcvs.2014.06.007
Many options exist for obtaining samples from the parenchymal lung nodule for pathologic analysis, including transthoracic image-guided needle aspiration (TTNA), transbronchial biopsy (TBNA), endobronchial ultrasoundguided biopsy, navigational bronchoscopic guidance biopsy, and thoracoscopic or open lung biopsy. Parenchymal pulmonary nodules located in proximity to the mediastinum, in front of the vertebral column, in proximity to major vessels, or behind the heart can be technically challenging and dangerous to biopsy using traditional image-guided techniques. Endoscopic ultrasound-guided fine needle aspiration (EUS-FNA) has become common for diagnosing mediastinal lymph nodes in lung cancer; however, its utility in biopsying parenchymal pulmonary nodules is not known. The present study reports on the largest reported experience with transesophageal needle aspiration of lung nodules using real-time EUS guidance.

\section{METHODS}

The study was a retrospective cohort study using a prospective interventional endoscopy research database. Patients presented to the CHUM Endoscopic Tracheobronchial and Oesophageal Center in the Division of Thoracic Surgery at the Centre Hospitalier de l'Universite de Montréal during a 31-month period with a lung mass suspicious for either a primary or secondary neoplasm of the lung. These patients had undergone attempts at lung biopsy using traditional techniques that were unsuccessful at obtaining a diagnosis, were not eligible for traditional lung nodule 


\section{Abbreviations and Acronyms \\ CT = computed tomography \\ EUS = endoscopic ultrasound \\ EUS-FNA $=$ endoscopic ultrasound-guided fine needle aspiration \\ NSCLC $=$ non-small cell lung cancer \\ TBNA $=$ transbronchial biopsy \\ TTNA $=$ transthoracic image-guided needle aspiration}

biopsy owing to anatomy or nodule location, or traditional techniques for lung nodule biopsy were thought to be too dangerous to attempt. All patients undergoing transesophageal biopsy of an intraparenchymal lung lesion using real-time EUS during the study period were included. The aim of the present study was to evaluate the rate of successful tissue sampling, the accuracy of the diagnostic test, and safety.

The institutional review board at the Research Center of the Centre Hospitalier de l'Université de Montréal approved the electronic prospective database used for the present study and approved this trial. Patient consent was obtained for entry into the prospective database; the institutional review board granted a waiver for individual patient consent for the present retrospective review.

All patients had undergone a standard diagnostic evaluation of their lung lesion with a history, physical examination, and standard imaging studies, including $\mathrm{CT}$ of the chest and upper abdomen with intravenous contrast and 5-mm cuts. Positron emission tomography scans were performed in patients with a suspicion for NSCLC. Positron emission tomography was performed from the skull base to the mid-thigh level. The maximum standardized uptake value of the primary and each suspicious lymph node station was determined. The clinical TNM stage was recorded. In certain patients, a biopsy was desirable for treatment planning. Such patients included those with a history of a treated malignancy, multiple comorbidities who were at high risk for lung resection, and those considered for stereotactic body radiotherapy. In addition, patients who met the criteria for invasive mediastinal staging according to the most recent American College of Chest Physician's guidelines were included if an EUS-guided lung biopsy had been undertaken in conjunction with their staging procedure. ${ }^{3}$ Patients with an endobronchial component were excluded, because biopsy of the lesion was undertaken transbronchially. The data from all patients were reviewed for the possibility of a transthoracic or transbronchial needle biopsy by radiologists, pulmonologists, and thoracic surgeons. For all patients, these procedures had either been attempted and found to be nondiagnostic or were not deemed safe owing to the anatomy.

\section{EUS Technique}

A convex probe EUS scope was used to perform real-time transesophageal needle biopsy (EUS Linear Scope, GF-UC140P-AL5; Olympus America Inc, Center Valley, Pa). A dedicated 22-gauge needle (ECHO-122; Cook Medical Ireland Ltd, Limerick, Ireland) was used to perform all EUS needle biopsy procedures under real-time ultrasound guidance. No core needle biopsies were used. No transvascular biopsies were performed. The smears were air dried and fixed on a slide. Additional samples were preserved in Cytolyt solution for cell block preparation. We performed as many passes as needed until satisfied with the gross appearance of the specimen. On average, this required 2 to 4 passes. Rapid on-site evaluation by a cytopathologist was not performed. An upright chest radiograph was obtained in the recovery area after the procedure to rule out iatrogenic pneumothorax. Conscious sedation was used for all procedures.

\section{Definitions and Statistical Methods}

A negative result was defined as a lung nodule labeled as benign by EUS and an adequate needle aspiration that identified the absence of tumor cells. A false-negative result was defined as the subsequent identification of malignancy, by either resection or biopsy using another method, within a mass deemed negative for malignancy by EUS-FNA. The efficacies (accuracy, sensitivity, and negative predictive value) of these tests were computed using standard definitions.

\section{RESULTS}

From October 2010 to May 2013, 1165 patients underwent combined endosonographic evaluation of biopsy-proven lung cancer or highly suspicious pulmonary lesions using combined endobronchial and endoesophageal ultrasound. Of the 1165 patients, 55 underwent planned transesophageal real-time ultrasound-guided biopsy of a suspicious lung lesion. The demographic data for these patients are listed in Table 1. The remaining 1110 patients did not undergo transesophageal lung biopsy. Therefore, no patient had transesophageal biopsy planned that was not attempted owing to anatomic constraints. All patients underwent $\mathrm{CT}$ of the chest and upper abdomen with $\geq 5$-mm collimated cuts.

In all patients, the lesion was visualized using ultrasonography and correlated with the suspicious lesion on the CT scan. The distribution of the location for all the lesions is listed in Table 1, with the right and left upper lobes the most common location for the lesions in the present series. Unsurprisingly, all the lesions were located within the inner third of the thoracic cavity, making access by way of the esophagus feasible.

A diagnostic sample was obtained from 52 of the 55 patients, for a diagnostic yield of $94.5 \%$. In all patients with adequate biopsy sampling, an accurate pathocytologic diagnosis of the target parenchymal nodules was obtained. The accuracy and sensitivity of EUS-FNA were both $94.5 \%$ and was consistent with the diagnosis on pathologic resection or clinical disease progression, or both. The distribution of the diagnoses is listed in Table 2. NSCLC was the most common diagnosis in the present series. In all 3 patients with a nondiagnostic sample, additional pathologic analysis was obtained in the form of anatomic lung resection ( 2 pneumonectomy and 1 lobectomy). In all 3 patients, the final pathologic examination confirmed the presence of NSCLC. No negative results were found, and all diagnostic results yielded a diagnosis of malignancy. Therefore, the prevalence of malignant disease in the present series was $100 \%$.

The nodule volume on EUS compared with that on CT scans had a Pearson product-moment correlation coefficient of 0.954 and Student $t$ test result of 0.044. The diagnostic accuracy was unaffected by tumor phenotype between NSCLC and small cell lung cancer.

No patients had either pneumothorax or new pleural effusion on the postprocedural chest radiograph. No immediate 
TABLE 1. Demographic and preprocedure data

\begin{tabular}{lc}
\hline \multicolumn{1}{c}{ Variable } & Value \\
\hline Age (y) & 63 \\
Mean & $39-83$ \\
Range & $31(56)$ \\
Female sex (n) & \\
Largest dimension on CT (mm) & 49.3 \\
Mean & $8-110$ \\
Range & $2(3.6)$ \\
$<15$ & $10(18)$ \\
$16-30$ & $43(78)$ \\
$>30$ & \\
Distance between lesion and esophagus (mm) & 25 \\
Mean & $0-46$ \\
Range & 56 \\
Patients who underwent PET (\%) & \\
Location & $23(42)$ \\
Right upper lobe & $10(18)$ \\
Right lower lobe & $16(29)$ \\
Left upper lobe & $6(11)$ \\
Left lower lobe & \\
\hline Data presented as $\mathrm{n}(\%)$, unless otherwise noted. CT, Computed tomography; \\
PET, positron emission tomography.
\end{tabular}

or 30-day complications related to the EUS lung biopsy procedure occurred. All patients were discharged on the same day of their procedure. No readmissions were required for any procedure-related complications. No patients reported dysphagia or odynophagia on long-term follow-up.

\section{DISCUSSION}

With the advent of lung cancer screening, the incidence of lung nodules and masses requiring evaluation has increased. In the present study, we have demonstrated the utility of transesophageal ultrasound-guided lung biopsy in establishing a cytopathologic diagnosis in a much selected group of patients requiring biopsy for whom anatomic constraints make traditional methods unfeasible. We report a diagnostic yield of $>90 \%$, albeit in a selected group of patients with a disease prevalence of $100 \%$.

Regardless of stage, the goal of the initial evaluation is to confirm the diagnosis and establish the disease stage using tests with the greatest accuracy and lowest associated morbidity. ${ }^{4}$ The lung nodule biopsy options include TTNA, TBNA, endobronchial ultrasound-guided biopsy, navigational bronchoscopic guidance biopsy, and thoracoscopic

TABLE 2. Results of needle biopsy

\begin{tabular}{lc}
\hline \multicolumn{1}{c}{ Diagnosis } & Patients (n) \\
\hline Non-small cell lung cancer & $44(80)$ \\
Small cell lung cancer & $4(7.3)$ \\
Metastatic neoplasm & $4(7.3)$ \\
Nondiagnostic sample & $3(5.5)$ \\
\hline
\end{tabular}

Data in parentheses are percentages. or open lung biopsy. The choice of technique is largely dependent on the location of the lesion.

For peripheral lesions, TTNA has been reported to be safe and efficacious and is the procedure of choice for biopsy of lung lesions in this subset of patients. Geraghty and colleagues $^{5}$ reported their experience with TTNA in 846 patients, with a sensitivity of $91 \%$ and specificity of $99 \%$. The incidence of pneumothorax in their series was $38 \% .^{5}$ This percentage was related partially to needle size, and they concluded that the use of a coaxial 18-gauge needle might reduce the incidence of this complication without compromising the diagnostic accuracy. ${ }^{5}$ However, studies on the efficacy of TTNA have been limited to reports of peripheral lung lesion. Although few data have been published regarding its role in central or hilar lesions, it only seems natural that the test performance could be compromised. For such central lesions, TBNA has been the preferred approach. Multiple options exist, including direct TBNA, brush cytology, bronchoalveolar lavage, ultrasound-guided and radial probe endobronchial ultrasound, and, more recently, electromagnetic navigational bronchoscopy. The reported sensitivity of TBNA in the setting of a visible endobronchial abnormality has been $88 \%{ }^{6}$ However, that study included patients with an endobronchial abnormality, whether a visible intraluminal lesion or extrinsic compression of a bronchus. Baaklini and colleagues ${ }^{7}$ examined the accuracy of TBNA techniques in relation to the distance from a central bronchus. They noted that the sensitivity of TBNA varied with the distance from the hilum. ${ }^{7}$ The yield of bronchoscopy in central, intermediate, and peripherally located lesions was $82 \%, 61 \%$, and $53 \%$, respectively. Thus, TBNA is preferred for central, peribronchial lesions, especially if an endobronchial component is found.

In certain cases, a suspicious lesion might not be easily classified as a central or peripheral lesion. An example would be the lesions in the patients whose scans are shown in Figure 1. These lesions were located within the inner third of the thoracic cavity, out of reach of TTNA in most institutions. However, they were not in a peribronchial location amenable to TBNA. These were peripheral lesions located adjacent to the mediastinal surface of the lung, ideal for transesophageal biopsy. This is not a novel technique, and previous studies have reported the use of EUS for biopsy of lung lesions. Aaraya and colleagues ${ }^{8}$ reported on the use of transesophageal needle biopsy for the molecular diagnosis of lung cancer. That study included 26 patients undergoing biopsy. Most of the patients underwent biopsy of mediastinal lesions; however, 5 patients underwent biopsy of pulmonary nodules without complications. The diagnostic yield was $100 \%$ for lung cancer using combined transesophageal and bronchoscopic techniques, with an additional diagnostic gain of $69 \%$ for EUS and bronchoscopy compared with bronchoscopy 


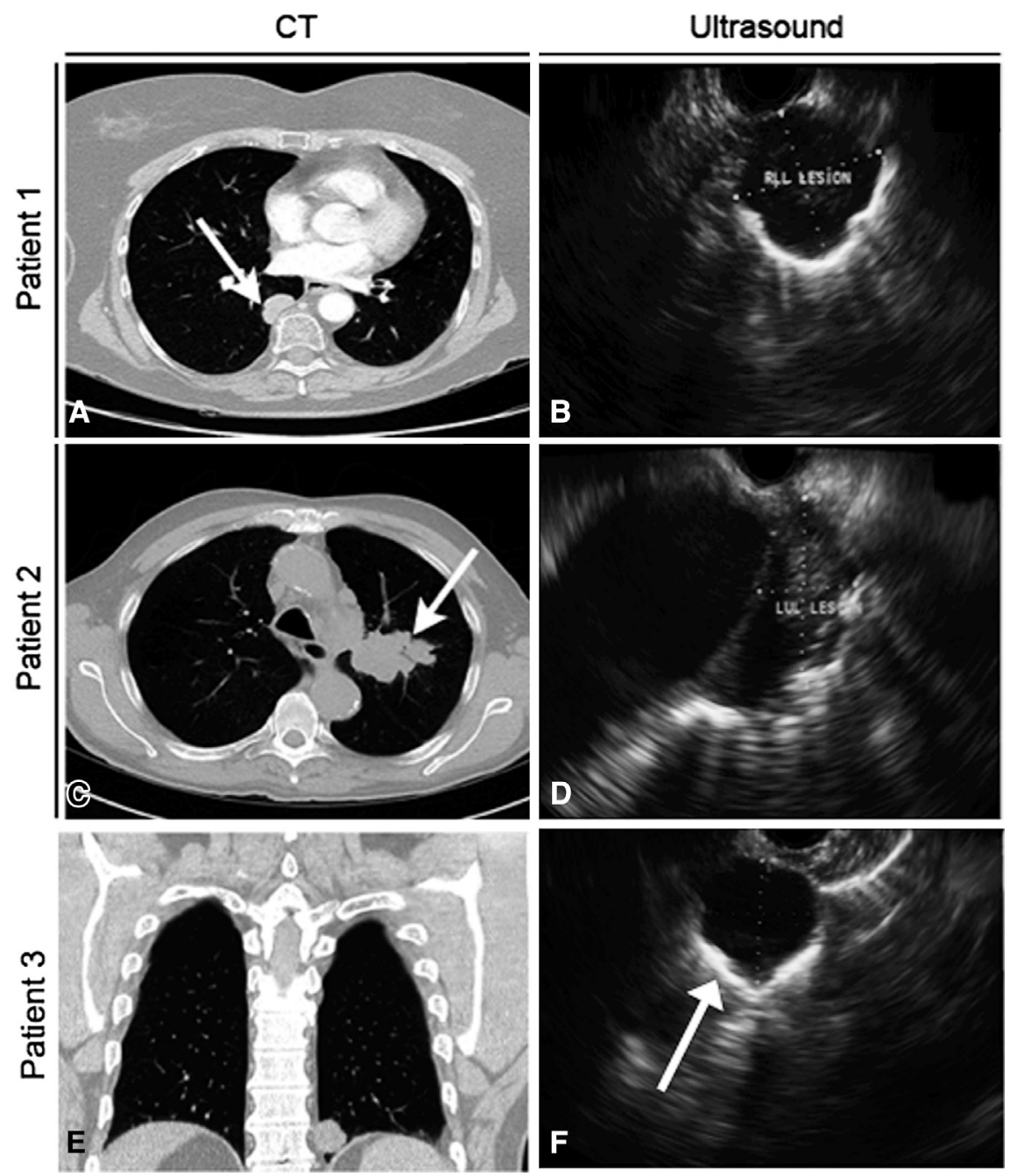

FIGURE 1. A, C, and E, Images of computed tomography scans and (B), (D), and (F), corresponding endoscopic ultrasound images. These were patients who had undergone a failed attempt at transbronchial needle biopsy (patient 2) or were not candidates for either transbronchial or transthoracic needle biopsy (patients 1 and 3) for anatomic reasons. Arrows indicate abnormality. CT, Computed tomography.

alone. Another study, by Bugalho and colleagues, ${ }^{9}$ demonstrated the efficacy of transesophageal EUS in the diagnosis of lung lesions in 51 patients who had undergone a nondiagnostic attempt using TTNA or TBNA. The sensitivity, specificity, and negative predictive value of combined transbronchial and transesophageal ultrasound needle biopsy was $89.8 \%, 100 \%$, and $20 \%$, respectively. Similar to our study, theirs was a highly selected group of patients with a prevalence of malignancy of $97.5 \%$ and a mean lesion size of $32.1 \mathrm{~mm}$. Two additional reports have been published of transesophageal EUS-guided lung biopsy using a dedicated EUS scope. The first, by Annema and colleagues, ${ }^{10}$ demonstrated a diagnostic yield of $97 \%$ in a group of 32 patients. The second study, by Varadarajulu and colleagues, ${ }^{11}$ reported on EUS-guided needle biopsy of lung lesions after unsuccessful attempts at CT-guided or bronchoscopic sampling to establish a tissue diagnosis.
They demonstrated a $100 \%$ diagnostic yield in 18 patients. $^{11}$

As we have demonstrated in our series, this technique is safe and yields a diagnosis for $95 \%$ of cases. In the 3 patients in whom a diagnosis was not obtained, the result was inconclusive, not falsely negative. This compares very well to contemporary reports of the TTNA and TBNA techniques. Furthermore, recent data have shown that EUS mediastinal staging is at least as good as surgical staging using mediastinoscopy. ${ }^{12,13}$ The addition of a EUS-guided lung biopsy at the same time during combined endosonographic staging seems logical. With a positive result, this could eliminate the need for an additional procedure such as TTNA. A theoretical risk exists of tumor seeding along the needle track. It is difficult to comment on such an incidence with the small number of patients and limited long-term follow-up. Anecdotally, however, we 
have not found any cases in which tumor seeding along the needle track resulted in a change in stage or oncologic outcome.

A comment should be made about the analysis of positive results. The reference standard for pathologic diagnosis is surgical resection of the nodule. If a patient underwent resection, the positive biopsy result was confirmed by the final pathologic examination. However, in this cohort, surgical resection was not undertaken in all patients owing to an advanced stage or medical inoperability. In patients with advanced disease, disease progression was found at a median follow-up of 5 months. The most difficult group to treat was patients with early-stage tumors who were medically inoperable. In all these patients, a positive result by EUS was not confirmed and was assumed to be true.

The present study had several limitations. The most obvious was that the present series was a retrospective analysis with all the limitations inherent to such studies. Furthermore, the prevalence of malignant disease in our series was $100 \%$. This was a highly selected group of patients with large tumors and significant fluorodeoxyglucose avidity on positron emission tomography scans such that the performance of any test was likely to be good. This raises 3 issues. The first is whether it is necessary to confirm such masses with a biopsy because the pretest probability is overwhelmingly in favor of malignancy. We believe that biopsy is not necessary in cases that are resectable in low-risk patients, for whom the overwhelming likelihood is primary lung cancer. However, in patients with a high risk for resection or patients for whom neoadjuvant or definitive chemotherapy or radiotherapy is indicated, a biopsy is desirable. Another group of patients who will benefit from biopsy are those with a history of treated extrathoracic malignancy, for whom the question will be a metastatic lesion versus primary lung cancer. In these patients, the treatment plans will diverge significantly, and a biopsy will be helpful in planning care. The second issue is related to the first, insofar as that the cohort represents a highly selected group of patients with large tumors that were centrally located with a prevalence of malignancy of $100 \%$. Because this was our early experience, we selected patients with a high probability of success. Thus, it is difficult to define the role of EUS-guided lung biopsy for smaller lesions. With more experience with the technique, we hope that in the future we will publish our results with smaller or more difficult to access lesions. The third issue is how to define the role of EUS lung biopsy compared with TTNA and TBNA. This is a difficult question to answer, because our study was not a comparative study, and TTNA or TBNA could certainly have performed well in a subset of patients with a $100 \%$ disease prevalence. Rather, EUS is a complimentary technique that can be used to confirm disease in cases in which either TTNA or TBNA is not feasible. The patients in the present study had undergone nondiagnostic TTNA or TBNA or were not candidates for TTNA or TBNA owing to the tumor location or patient anatomy.

\section{CONCLUSIONS}

We believe that our results have demonstrated the feasibility and safety of EUS lung biopsy in patients with primary and secondary lung malignancies requiring a tissue diagnosis. At our institution, in cases in which a diagnosis is required before treatment, percutaneous transthoracic and transbronchial techniques remain the most common methods of obtaining tissue from lung nodules. We use EUS lung biopsy in selected patients in whom the lesion is adjacent to the mediastinum but not located peribronchially, such that transbronchial techniques are applicable. We have mainly used it in situations in which the pretreatment biopsy results will alter treatment, such as high-risk patients or patients with a history of a treated extrathoracic malignancy, and to differentiate small cell lung cancer from NSCLC. No complications developed in the present small series.

\section{References}

1. Siegel R, Ma J, Zou Z, Jemal A. Cancer statistics, 2014. CA Cancer J Clin. 2012; 64:9-29.

2. Aberle DR, Adams AM, Berg CD, Black WC, Clapp JD, Fagerstrom RM, et al. Reduced lung-cancer mortality with low-dose computed tomographic screening. N Engl J Med. 2011;365:395-409.

3. Silvestri GA, Gonzalez AV, Jantz MA, Margolis ML, Gould MK, Tanoue LT, et al. Methods for staging non-small cell lung cancer: diagnosis and management of lung cancer, 3rd ed: American College of Chest Physicians evidence-based clinical practice guidelines. Chest. 2013;143(5 Suppl):e211S-50S.

4. Gould MK, Donington J, Lynch WR, Mazzone PJ, Midthun DE, Naidich DP, et al. Evaluation of individuals with pulmonary nodules: when is it lung cancer? Diagnosis and management of lung cancer, 3rd ed: American College of Chest Physicians evidence-based clinical practice guidelines. Chest. 2013;143(5 Suppl):e93S-120S.

5. Geraghty PR, Kee ST, McFarlane G, Razavi MK, Sze DY, Dake MD. CT-guided transthoracic needle aspiration biopsy of pulmonary nodules: needle size and pneumothorax rate. Radiology. 2003;229:475-81.

6. Schreiber G, McCrory D. Performance characteristics of different modalities for diagnosis of suspected lung cancer. Chest. 2003;123(Suppl):115S-28S.

7. Baaklini WA, Reinoso MA, Gorin AB, Sharafkaneh A, Manian P. Diagnostic yield of fiberoptic bronchoscopy in evaluating solitary pulmonary nodules. Chest. 2000;117:1049-54.

8. Aaraya T, Demura Y, Kasahara K, Matsuoka H, Yamamura K, Nishitsuji M, et al. Usefulness of transesophageal bronchoscopic ultrasound-guided fine-needle aspiration in the pathologic and molecular diagnosis of lung cancer and lesions adjacent to the esophagus. J Bronchol Interv Pulmonol. 2013;20:121-6.

9. Bugalho A, Ferreira D, Eberhardt R, Dias SS, Videira PA, Herth FJ, et al. Diagnostic value of endobronchial and endoscopic ultrasound-guided fine needle aspiration for accessible lung cancer lesions after non-diagnostic conventional techniques: a prospective study. BMC Cancer. 2013;19:130.

10. Annema JT, Veseliç M, Rabe KF. EUS-guided FNA of centrally located lung tumours following a non-diagnostic bronchoscopy. Lung Cancer. 2005; 48:357-61.

11. Varadarajulu S, Hoffman BJ, Hawes RH, Eloubeidi MA. EUS-guided FNA of lung masses adjacent to or abutting the esophagus after unrevealing CT-guided biopsy or bronchoscopy. Gastrointest Endosc. 2004;60:293-7.

12. Yasufuku K, Pierre A, Darling G, de Perrot M, Waddell T, Johnston M, et al. A prospective controlled trial of endobronchial ultrasound-guided transbronchial needle aspiration compared with mediastinoscopy for mediastinal lymph node staging of lung cancer. J Thorac Cardiovasc Surg. 2011;142:1393-400. 
13. Liberman M, Sampalis J, Duranceau A, Thiffault V, Hadjeres R, Ferraro P. Endosonographic mediastinal lymph node staging of lung cancer. Chest. March 6, 2014 [Epub ahead of print]

\section{Discussion}

Dr Kenton J. Zehr (Baltimore, Md). How user dependent is this technique? Do you think everybody can do this, or does this require technical skill beyond the norm?

Dr Liberman. I would say that it depends on the size of the nodule. For the 8-mm nodule, it might require more technical savvy or experience. However, for the larger lesions, this is not very complex. It is fast. It takes about 30 seconds to 1 minute. Most of these procedures can be done with local sedation and without general anesthesia as an outpatient procedure, and I would say that anyone doing EUS for mediastinal lymph nodes can do this without a lot of practice.

Dr Zehr. Do you think this approach for diagnosis will become part of the guidelines?

Dr Liberman. I would say that in general we do not recommend biopsying all lung nodules before surgery in low-risk patients who have a high likelihood of malignancy. I think the usefulness of this procedure, which is similar to that for transthoracic needle aspiration, is in the high-risk patient or a patient in whom the diagnosis will change your surgical management.

Also, I would say that, as most people know, the complication and pneumothorax rate of a TTNA under CT guidance ranges from $10 \%$ to $20 \%$, depending on which center and the severity of the emphysema, and we have seen no pneumothorax, even in high-risk patients with emphysema.

Dr Vinay Badhwar (Pittsburgh, Pa). The technology of EUS-FNA is still in the process of development. Do you think for distant lesions or lesions that are just beyond the scope, additional technologic ultrasound modification should be required, such as the use of 3-dimensional ultrasound?

Dr Liberman. It is a good question. Air is the enemy of ultrasound, and ultrasonographers do not like air, and the lung is full of air. So I think that makes it very, very hard.

As the lesions get further away from the mediastinum or from your target, which is your EUS scope, it becomes more difficult to see these lesions. Ultrasound technology on EUS compared with endobronchial ultrasound has been around for almost 20 years now, and the technology is in its 10th or 12th generation. Also, we are pretty good at seeing these lesions.

Again, I think as technology improves, it will become easier and easier to use these devices. However, we see these nodules quite well. 